\title{
IDEAL LIOUVILLE DOMAINS A cool gadget
}

\author{
Emmanuel GIROUX* \\ Montreal — August 2017
}

The purpose of these notes is to describe a convenient packaging for those objects nowadays called Liouville domains but which have been studied formerly under various names such as "symplectic manifolds with restricted contact type boundaries" or "complete convex symplectic manifolds with conical/cylindrical ends" [We, EG, $\mathrm{Mc}, \mathrm{Ge}, \mathrm{La}, \mathrm{CFH}, \mathrm{Vi}, \mathrm{Se}, \mathrm{CE}]$. In this text, the term domain systematically refers to a compact manifold with boundary.

Definition 0 (Liouville Domains). A Liouville domain is a domain $F$ endowed with a Liouville form, that is, a 1-form $\lambda$ satisfying the following two axioms:

- $\omega:=d \lambda$ is a symplectic form on $F$.

- $\lambda$ induces a contact form on $K:=\partial F$ orienting $K$ as the boundary of $(F, \omega)$.

Liouville domains are ubiquitous in symplectic geometry. Obvious examples are starshaped tubes about the zero-section in cotangent spaces of closed manifolds. More generally, Stein domains are fundamental examples. In addition, it follows from Donaldson's work [Do] that every closed integral symplectic manifold can be obtained from a Liouville domain (whose Reeb flow on the boundary defines a free circle action) by a "symplectic reduction" crashing the boundary to its quotient by the circle action. Similarly, every closed contact manifold can be constructed from a Liouville domain as the "relative mapping torus" of some symplectic self-diffeomorphism fixing the boundary points [Gi1].

\footnotetext{
${ }^{*}$ Centre National de la Recherche Scientifique (UMI 3457 CRM) and Université de Montréal.
} Partially supported by the ANR grant MICROLOCAL (ANR-15CE40-0006). 
On each Liouville domain, there is a wide (open) choice of Liouville forms. In particular, any Liouville form $\lambda$ has many multiples $e^{f} \lambda$ which are Liouville forms as well: The pertinent condition on the function $f$ is that $\lambda \cdot f>-1$, the Liouville vector field $\lambda$ being given by $\lambda\lrcorner d \lambda=\lambda$. By the second axiom of Definition 0 , $\lambda$ points transversely outwards along $\partial F$ (because $\lambda\lrcorner(d \lambda)^{n}=n \lambda \wedge(d \lambda)^{n-1}$ ), so the inequality $\lambda>-1$ admits a big convex set of solutions $f$. However, these rescaled Liouville forms share important geometric features: They determine the same contact structure $\xi$ (with all possible contact forms) on $\partial F$, the singular foliations spanned by their Liouville fields coincide, and the symplectic structures they define on $F$ are the same up to completion (and sliding in the direction of the Liouville fields). The completion of a Liouville domain $(F, \lambda)$ is an open manifold $\widehat{F} \supset F$ equipped with a 1 -form $\widehat{\lambda}$ such that:

- $\widehat{\omega}:=d \widehat{\lambda}$ is a symplectic form on $\widehat{F}$,

- $\widehat{\lambda} \uparrow_{F}$ equals $\lambda$, and

- $\lambda$ is a complete vector field whose flow induces a diffeomorphism from $\partial F \times \mathbb{R}_{\geq 0}$ to $\widehat{F}-\operatorname{Int} F$.

It is easy to check that such a completion always exists and is unique up to symplectomorphism. More precisely, between any two completions of the same domain $(F, \lambda)$, there is a unique diffeomorphism which is the identity on $F$ and conjugates the extended Liouville forms.

The completion $(\widehat{F}, \widehat{\lambda})$ offers an alternative description of the contact manifold $(\partial F, \xi)$ (with no preferred contact form) as the orbit space of $\lambda$ at infinity. In [EKP], this orbit space is called the ideal contact boundary of $(\widehat{F}, \widehat{\lambda})$. A natural question then arises: Does this ideal contact boundary really depend on the form $\widehat{\lambda}$ (with well-behaved dual vector field) or only on the symplectic form $\widehat{\omega}:=d \widehat{\lambda}$ ? This question was studied by S. Courte in his thesis and he exhibited examples of completions with isomorphic symplectic structures but non-diffeomorphic ideal contact boundaries [Co1, $\mathrm{Co} 2]$. The observation leading to the concept of ideal Liouville domains is that this ambiguity about the ideal contact boundary can be lifted by fixing a smooth compactification of the completion "taming" the symplectic structure. Ideal Liouville domains are domains with a symplectic structure in the interior (subject to some "tameness condition" near the boundary) which uniquely determines a contact structure on the boundary. They have an affine space of (ideal) Liouville forms (and none of them being part of the data) 
whose dual vector fields are complete and hit the boundary transversely. They enjoy the stability that is expected from symplectic objects (for the suitable topology) and they can be manipulated (modified and combined) by means of various operations, notably products (without corners), handle attachments (in particular, boundary connect sums), and plumbings (along proper Lagrangian disks with Legendrian boundaries). They are also very useful to understand and describe the relationships between contact structures and open books, and they were actually introduced in this context. The second half of the paper is devoted to the contact aspects. After discussing subtleties related to the monodromy of open books, we define and study Liouville open books. We prove that every open book supporting a contact structure is a Liouville open book and that every Liouville open book supports an essentially unique contact structure.

Acknowledgments. I am indebted to Robert Roussarie for his nice observation reproduced in Remark 12, I also wish to thank Sylvain Courte for his helpful comments on the preliminary version of this text he used to write his thesis [Co2]. Finally, I am grateful to Patrick Massot and Klaus Niederkrüger for adopting and advertising the notion of ideal Liouville domains, and for pushing me to write these notes.

\section{A Ideal Liouville domains in their own}

Definition 1 (Ideal Liouville Domains). An ideal Liouville domain $(F, \omega)$ is a domain $F$ endowed with an ideal Liouville structure $\omega$. This ideal Liouville structure is an exact symplectic form on Int $F$ admitting a primitive $\lambda$ such that: For some (and then any) function $u: F \rightarrow \mathbb{R}_{\geq 0}$ with regular level set $\partial F=\{u=0\}$, the product $u \lambda$ extends to a smooth 1 -form on $F$ which induces a contact form on $\partial F$.

A 1-form $\lambda$ as above is called an ideal Liouville form. Its dual vector field $\lambda$ is an ideal Liouville field.

Liouville forms in ideal Liouville domains are analogous to contact forms on contact manifolds: They exist, and it is sometimes useful to choose one, but this choice is most often unimportant. In this analogy, ideal Liouville fields correspond to Reeb fields though their dynamics are very different: The latter are Hamiltonian while the former expand the symplectic form - and hence the volume exponentially. Speaking of contact manifolds, one of the striking features of ideal Liouville domains is: 
Proposition 2 (The Boundary Contact Structure). Let $(F, \omega)$ be an ideal Liouville domain. Then the boundary $K:=\partial F$ has a positive contact structure $\xi$, uniquely determined by $\omega$, which is left invariant by any diffeomorphism of $F$ preserving $\omega$. Moreover, the positive equations of $\xi$ are in one-to-one correspondence with the negative sections of the conormal bundle of $K$.

As a consequence of the latter claim, every symplectic diffeomorphism $\phi$ of $(F, \omega)$ (meaning a diffeomorphism of $F$ whose restriction to the interior preserves $\omega$ ) which is relative to the boundary (in the sense that its restriction to $\partial F$ is the identity) is actually tangent to the identity at all points of $\partial F$. Indeed, $\phi$ preserves the equations of the boundary contact structure and hence acts trivially on the conormal bundle of $\partial F$.

Proof. Let $\lambda$ be a Liouville form and $u: F \rightarrow \mathbb{R}_{\geq 0}$ a function with regular level set $K=\{u=0\}$. By assumption, $u \lambda$ extends to a smooth 1-form $\beta$ on $F$ which induces a contact form on $K$. Write

$$
\omega=d \lambda=d(\beta / u)=u^{-2}(u d \beta+\beta \wedge d u) .
$$

This formula demonstrates that $u^{2} \omega$ extends to a smooth 2-form $\gamma$ on $F$ which depends on $u$ only up to a conformal factor. Now, along $K$, the form $\gamma$ has rank 2 , and its kernel is the contact structure $\xi$ on $K$ defined by $\beta$. It follows that $\xi$ is independent of the choice of $\lambda$ and $u$. Moreover, the identity $\gamma=\beta \wedge d u$ shows that $\left.\beta\right|_{K}$ is also independent of $\lambda$ and is uniquely (and pointwise linearly) determined by $d u$ viewed as a section of the conormal bundle of $K$.

Now recall that the symplectization of a contact manifold $(K, \xi)$ is the symplectic submanifold $S K$ of $T^{*} K$ consisting of non-zero covectors $\beta_{p} \in T_{p}^{*} K$, $p \in K$, whose cooriented kernel is $\xi_{p}$ (contact structures are cooriented in this text). We denote by $\lambda_{\xi}$ the 1 -form induced on $S K$ by the canonical Liouville form of $T^{*} K$. We also define the "projective completion" of $S K$ as the quotient

$$
\overline{S K}:=\left(S K \times \mathbb{R}_{\geq 0}\right) / \mathbb{R}_{>0}
$$

where $\mathbb{R}_{>0}$ acts (freely, properly and) diagonally by multiplication. Thus, $\overline{S K}$ is a smooth manifold with boundary obtained by attaching a copy of $K=S K / \mathbb{R}_{>0}$ to $S K=\left(S K \times \mathbb{R}_{>0}\right) / \mathbb{R}_{>0}$.

Proposition 3 (Ideal Liouville Fields). Let $(F, \omega)$ be an ideal Liouville domain, $(K, \xi)$ its contact boundary, and $\lambda$ an ideal Liouville form in $\operatorname{Int} F$. 
a) The Liouville field $\lambda$ is complete and the singular foliation spanned by $\lambda$ extends to a foliation of $\vec{F}$ which is non-singular along $K$ and transverse to $\vec{K}$. We denote by $U$ the open collar neighborhood of $K$ consisting of all extended leaves reaching $K$.

b) There exists a unique embedding $\iota=\iota_{\lambda}: \overline{S K} \rightarrow F$ such that $\iota \uparrow_{K}=$ id and $\iota^{*} \lambda=\lambda_{\xi}$; its image is the open collar neighborhood $U$.

Proof. Let $u: F \rightarrow \mathbb{R}_{\geq 0}$ be a function with regular level set $K=\{u=0\}$ and $\beta$ the form extending $u \lambda$ over $F$. For $n=\frac{1}{2} \operatorname{dim} F$,

$$
\begin{aligned}
\omega^{n}=(d(\beta / u))^{n}=u^{-2 n}(u & d \beta+\beta \wedge d u)^{n} \\
& =u^{-n-1}(u d \beta+n \beta \wedge d u) \wedge(d \beta)^{n-1}=u^{-n-1} \mu
\end{aligned}
$$

where $\mu:=(u d \beta+n \beta \wedge d u) \wedge(d \beta)^{n-1}$ is a volume form on $F$.

Let $\nu$ denote the vector field on $F$ given by $\nu\lrcorner \mu=n \beta \wedge(d \beta)^{n-1}$. Since $\beta$ induces a positive contact form on the boundary, $\nu$ is non-singular along $K$ and points transversely outwards (specifically, $\nu \cdot u=-1$ by the very definition of things). On the other hand, in the interior of $F$,

$$
\left.\left.n \beta \wedge(d \beta)^{n-1}=n u^{n} \lambda \wedge(d \lambda)^{n-1}=u^{n} \underline{\lambda}\right\lrcorner \omega^{n}=u^{-1}(\underline{\lambda}\lrcorner \mu\right) .
$$

Comparing this relation with the definition of $\nu$, we get $\lambda=u \nu$. Since $u$ vanishes along $K$, the vector field $\lambda$ is complete and the foliation it defines extends to the foliation spanned by $\nu$. This proves Part a).

As for Part b), first note that if the embedding $\iota$ exists then it maps the standard Liouville field $\lambda_{\xi}$ of $S K$ to $\vec{\lambda}$, and so its image has to be $U$. Now observe that the holonomy of the foliation spanned by $\nu$ yields a projection $U \rightarrow K$ and, for any point $p \in U-K$ projecting to $q \in K$, identifies $\lambda_{p} \in T_{p}^{*} U$ with a covector in $T_{q}^{*} K$ whose cooriented kernel equals $\xi_{q}$ (just because the holonomy preserves the kernel of $\beta=u \lambda$ ). Thus, we have a smooth map $U \rightarrow \overline{S K}$ which is the identity on $K$. The expansion properties of the flow of $\lambda$ imply that this map is a diffeomorphism, and we define $\iota$ to be the inverse map. The relation $\iota^{*} \lambda=\lambda_{\xi}$ follows from the very definition of $\lambda_{\xi}$, and $\iota$ is unique because the identity of $K$ lifts to a unique diffeomorphism of $S K$ preserving $\lambda_{\xi}$.

Corollary 4 (Ideal Liouville Forms). On any ideal Liouville domain $(F, \omega)$, ideal Liouville forms constitute an affine space. Given a function $u: F \rightarrow \mathbb{R}_{\geq 0}$ with regular level set $\partial F=\{u=0\}$, the underlying vector space can be described as consisting of all closed 1-forms $\kappa$ on Int $F$ satisfying the following equivalent conditions: 
(i) The form uк extends to a smooth form on $F$.

(ii) The vector field $\underline{\kappa_{g}} / u$ extends to a smooth vector field on $F$ (which is automatically tangent to $K:=\partial F$ ).

(iii) There exists a function $f: F \rightarrow \mathbb{R}$ such that $\kappa-d(f \log u)$ is the restriction of a closed 1-form on $F$.

As a result, a Lagrangian submanifold $L \subset \operatorname{Int} F$ is exact for some ideal Liouville form if and only if its Liouville class (with respect to an arbitrary given ideal Liouville form) lies in the image of the natural map $H^{1}(F, \mathbb{R}) \rightarrow H^{1}(L, \mathbb{R})$.

Proof. The only (maybe) non-trivial claim which is not a straightforward consequence of Propositions 2 and 3 is that (i) implies (iii). So assume that $u \kappa$ extends to a smooth form $\gamma$ on $F$. In Int $F$,

$$
0=d \kappa=d(\gamma / u)=u^{-2}(u d \gamma+\gamma \wedge d u) .
$$

By continuity, $u d \gamma+\gamma \wedge d u$ is identically zero on $F$, and hence $\gamma \wedge d u=0$ along $K=\{u=0\}$. Thus, there exists a function $f: K \rightarrow \mathbb{R}$ such that $\gamma=f d u$ along $K$. Extend $f$ (keeping its name) to a function on $F$ and observe that the form

$$
\gamma-u d(f \log u)=\gamma-u \log u d f-f d u
$$

extends to a 1-form $\gamma^{\prime}$ on $F$ which vanishes identically along $K$. It follows that $\gamma^{\prime}=u \kappa^{\prime}$ where $\kappa^{\prime}$ is a closed 1-form on $F$.

Another corollary is the following avatar of a standard lemma (see Lemma 1.1 and the subsequent remark in [BEE]):

Corollary 5 (Exact Isotopies). Let $(F, \omega)$ be an ideal Liouville domain and $\lambda_{t}$ $(t \in[0,1])$ a path of ideal Liouville forms in $\operatorname{Int} F$. Then there is a symplectic isotopy $\psi_{t}(t \in[0,1])$ of $F$, relative to the boundary, such that $\psi_{0}=\mathrm{id}$ and, for every $t \in[0,1]$, the form $\psi_{t}^{*} \lambda_{t}-\lambda_{0}$ is the differential of a function with compact support in Int $F$.

Here the path $\lambda_{t}$ is assumed to be smooth in the sense that $\lambda_{t}=\beta_{t} / u$ where $\beta_{t}$ $(t \in[0,1])$ is a smooth path of 1 -forms on $F$, ie. a smooth 1 -form on $[0,1] \times F$ whose contraction with $\partial_{t}$ is zero ( $u$, as usual, is a non-negative function on $F$ with regular level set $K:=\partial F=\{u=0\})$. 
Proof. For $t \in[0,1]$, let $\iota_{t}: \overline{S K} \rightarrow F$ be the unique embedding such that $\left.\iota_{t}\right|_{K}=\mathrm{id}$ and $\iota_{t}^{*} \lambda_{t}=\lambda_{\xi}$, where $\xi$ is the boundary contact structure (cf. Proposition 3). Setting $U_{t}:=\iota_{t}(\overline{S K})$, we have an isotopy of embeddings

$$
\psi_{t}^{0}:=\iota_{t} \circ \iota_{0}^{-1}: U:=U_{0} \rightarrow F
$$

with the following properties:

- $\psi_{0}^{0}=\mathrm{id}$ and $\psi_{t}^{0} \uparrow_{K}=\mathrm{id}$ for all $t \in[0,1]$,

- $\left(\psi_{t}^{0}\right)^{*} \lambda_{t}=\lambda_{0}$ on $U-K$ for all $t \in[0,1]$.

Therefore, the time-dependent vector field $\eta_{t}^{0}$ on $U_{t}$ generating the isotopy $\psi_{t}^{0}$ satisfies, for all $t \in[0,1]$,

- $\eta_{t}^{0}=0$ along $K$, and

- $\left(\eta_{t}^{0} \cdot \lambda_{t}\right)+\dot{\lambda}_{t}=0$ on $U_{t}-K$.

Let $f_{t}^{0}:=\lambda_{t}\left(\eta_{t}^{0}\right)$ and denote by $\eta_{t}^{1}$ the time-dependent locally Hamiltonian vector field on Int $F$ given by $\left.\eta_{t}^{1}\right\lrcorner \omega=-\dot{\lambda}_{t}$. In $U_{t}-K$,

$$
\left.\left(\eta_{t}^{1}-\eta_{t}^{0}\right)\right\lrcorner \omega=d f_{t}^{0} .
$$

Now take a time-dependent function $f_{t}$ on $F$ equal to $f_{t}^{0}$ near $K$, and consider the locally Hamiltonian vector field $\eta_{t}$ on Int $F$ such that $\left.\left(\eta_{t}^{1}-\eta_{t}\right)\right\lrcorner \omega=d f_{t}$. Since $\eta_{t}=\eta_{t}^{0}$ close to the boundary, $\eta_{t}$ extends smoothly to a vector field on $F$ which vanishes identically along $K$. On the other hand, in Int $F$,

$$
\left(\eta_{t} \cdot \lambda_{t}\right)+\dot{\lambda}_{t}=d\left(\lambda_{t}\left(\eta_{t}\right)-f_{t}\right)
$$

and the function $\lambda_{t}\left(\eta_{t}\right)-f_{t}$ is zero on the neighborhood of $K$ where $f_{t}=f_{t}^{0}$ (and $\eta_{t}=\eta_{t}^{0}$ ). The desired isotopy $\psi_{t}$ is obtained by integrating the vector field $\eta_{t}$.

Ideal Liouville domains are stable in the following sense:

Lemma 6 (Stability). Let $F$ be a domain and $\left(\omega_{t}\right)(t \in[0,1])$ a path of ideal Liouville structures on $F$. Then there exists an isotopy $\phi_{t}(t \in[0,1])$ of $F$ such that $\phi_{0}=\mathrm{id}$ and $\phi_{t}^{*} \omega_{t}=\omega_{0}$ for all $t \in[0,1]$. Moreover, we can choose this isotopy relative to $K=\partial F$ if - and clearly only if - all forms $\omega_{t}$ induce the same boundary contact structure. 
Here again, the required smoothness of the path $\omega_{t}$ is that there is a smooth path $\beta_{t}$ of 1 -forms on $F$ such that $\omega_{t}=d\left(\beta_{t} / u\right)$.

Sketch of proof. Due to the smoothness of the path $\omega_{t}$, the induced contact structure on $K$ vary smoothly with $t$. Then, by Gray's stability Theorem (and the obvious fact that any isotopy of $K$ extends to an isotopy of $F$ ), it suffices to treat the case when all forms $\omega_{t}$ induce the same boundary contact structure $\xi$. Using Proposition 3, we can further arrange that the forms $\omega_{t}$ coincide near $K$ and, more specifically, have smoothly varying ideal Liouville forms $\lambda_{t}$ which all agree in a neighborhood of $K$. Then we conclude with Moser's standard argument.

The next proposition is another expected and straightforward result relating the symplectic geometry of (the interior of) an ideal Liouville domain $(F, \omega)$ with the contact geometry of its boundary $(K, \xi)$. The notations are as follows:

- $\mathcal{D}(F, \omega)$ is the group of diffeomorphisms of $F$ preserving $\omega$,

- $\mathcal{D}_{\partial}(F, \omega) \subset \mathcal{D}(F, \omega)$ is the subgroup of diffeomorphisms fixing $K:=\partial F$ pointwise, and

- $\mathcal{D}(K, \xi)$ is the group of diffeomorphisms of $K$ preserving $\xi$.

Proposition 7 (Relations between Automorphism Groups). Let $(F, \omega)$ be an ideal Liouville domain with contact boundary $(K, \xi)$. The restriction homomorphism

$$
\mathcal{D}(F, \omega) \rightarrow \mathcal{D}(K, \xi)
$$

is a Serre fibration, with associated long exact sequence of homotopy groups

$$
\ldots \pi_{k} \mathcal{D}_{\partial}(F, \omega) \rightarrow \pi_{k} \mathcal{D}(F, \omega) \rightarrow \pi_{k} \mathcal{D}(K, \xi) \rightarrow \pi_{k-1} \mathcal{D}_{\partial}(F, \omega) \ldots
$$

The homomorphism $\pi_{1} \mathcal{D}(K, \xi) \rightarrow \pi_{0} \mathcal{D}_{\partial}(F, \omega)$ can be used to define natural semigroups in the symplectic mapping class group $\operatorname{MCG}(F, \omega):=\pi_{0} \mathcal{D}_{\partial}(F, \omega)$ : An element in there is positive (resp. non-negative) if it is the image of a positive (resp. non-negative) loop in $\mathcal{D}(K, \xi)$. When $(K, \xi)$ is a "contact circle bundle" (meaning that some Reeb flow generates a free circle action), the image of the corresponding loop is the mapping class of a "fibered Dehn twist".

Sketch of proof. We merely explain how to lift paths. Let $\phi_{0} \in \mathcal{D}(F, \omega)$ and take a path $\check{\phi}_{t} \in \mathcal{D}(K, \xi)(t \in[0,1])$ starting with $\check{\phi}_{0}=\phi_{0} \Upsilon_{K}$. The contact isotopy $\check{\phi}_{t}$ lifts to a Hamiltonian isotopy $S \check{\phi}_{t}$ in the symplectization $S K$. Pick an arbitrary 
ideal Liouville form $\lambda$ and identify $\overline{S K}$ with the collar neighborhood $U=\iota_{\lambda}(\overline{S K})$ of Proposition 3. The path $S \check{\phi}_{t}$ can then be viewed as a Hamiltonian isotopy of $U$ extending $\check{\phi}_{t}$. We obtain the path $\phi_{t}$ by cutting off the corresponding Hamiltonian functions away from $K$ inside $U$, and by integrating the new Hamiltonian functions with $\phi_{0}$ as the initial condition.

We now describe the two main examples of ideal Liouville domains.

Example 8 (in vivo: Convex Hypersurfaces in Contact Manifolds). Let $(V, \xi)$ be a contact manifold and $S$ a hypersurface in $V$ which is $\xi$-convex, meaning that $S$ is transverse to some contact vector field $\nu$. Consider the "dividing set"

$$
\Gamma:=\left\{p \in S: \nu_{p} \in \xi_{p}\right\} \subset S .
$$

Then the closure of every relatively compact connected component of $S-\Gamma$ is naturally an ideal Liouville domain (see [Gi1, I.3-C]).

To see this, pick an equation $\alpha$ of $\xi$, set $u:=\alpha(\nu)$ and note that $\Gamma$ is the zero-set of $u \uparrow_{S}$. We claim that $u \uparrow_{S}$ vanishes transversely. Indeed, the identity $\left.\left.d u\right|_{\xi}=-(\nu\lrcorner d \alpha\right) \uparrow_{\xi}$ (drawn from the Cartan formula for the Lie derivative) implies that $\left.d u\right|_{\xi \cap T S} \neq 0$ along $\Gamma$, and that $\Gamma$ is actually a contact submanifold of $(V, \xi)$. Moreover, $\nu$ restricted to the open set $\{u \neq 0\} \subset V$ is the Reeb vector field of the contact form $\alpha / u$. Since $\nu$ is transverse to $S$, the differential $d(\alpha / u)$ induces a symplectic form on $S-\Gamma$.

Example 9 (in vitro: Ideal Completion of a Liouville Domain). Let $(F, \lambda)$ be a Liouville domain in the sense of Definition 0 , and let $u: F \rightarrow \mathbb{R}_{\geq 0}$ be a function with the following properties:

- $u$ admits $K:=\partial F$ as its regular level set $\{u=0\}$,

- $\vec{\lambda} \cdot \log u<1$ at every point in Int $F$.

Then a simple calculation (already resorted to in the introduction) shows that $\omega:=d(\lambda / u)$ is a symplectic form on $\operatorname{Int} F$, and so $(F, \omega)$ is an ideal Liouville domain. Moreover, since conditions 1 and 2 define a convex cone of functions $u$, it follows from Lemma 6 that, up to isotopy relative to the boundary, the geometry of $(F, \omega)$ is independent of $u$. Taking $u$ non-increasing along the orbits of $\lambda$ and equal to 1 outside the collar neighborhood of $K$ associated with $\lambda$ (by Proposition 3 ), we see that $(\operatorname{Int} F, \omega)$ is symplectically isomorphic to the completion of $(F, \lambda)$. For this reason, $(F, \omega)$ is called the ideal completion of $(F, \lambda)$. It can be alternatively obtained by gluing $K$ to the usual completion $(\widehat{F}, \widehat{\lambda})$ in exactly the same way as $\overline{S K}$ was constructed from $S K$. 
To conclude this general discussion of ideal Liouville domains, here is the product construction alluded to in the introduction:

Proposition 10 (Product of Ideal Liouville Domains). Let $\left(F_{1}, \omega_{1}\right)$ and $\left(F_{2}, \omega_{2}\right)$ be two ideal Liouville domains. Up to isomorphism, there exists a unique ideal Liouville domain $(F, \omega)$ admitting a diffeomorphism $\phi: \operatorname{Int} F \rightarrow \operatorname{Int}\left(F_{1} \times F_{2}\right)$ such that $\omega=\phi^{*}\left(\omega_{1} \oplus \omega_{2}\right)$ and, for any Liouville forms $\lambda_{1}$ and $\lambda_{2}$ on $F_{1}$ and $F_{2}$, respectively, $\phi^{*}\left(\lambda_{1} \oplus \lambda_{2}\right)$ is a Liouville form on $F$.

Proof. Clearly, ( $\left.\operatorname{Int}\left(F_{1} \times F_{2}\right), \lambda_{1} \oplus \lambda_{2}\right)$ is the (usual) completion of some Liouville domain. The desired product is the ideal completion of this domain. Uniqueness follows from the convexity of the sets of ideal Liouville forms on $F_{1}$ and $F_{2}$.

Remark 11 (Generalizations). I presented the notion of ideal Liouville domains in a talk at ETH (Zurich) in November 2010 (for Eddi Zehnder's 70th birthday), and the first published paper where they explicitly appear is [MNW]. The concept was further generalized in [Co2] where Courte defined ideal Liouville cobordisms. A cobordism is an oriented domain $F$ whose boundary components are given prescribed orientations; $\partial_{+} F$ (resp. $\partial_{-} F$ ) denotes the union of the boundary components endowed with the boundary orientation (resp. with the reversed orientation). An ideal Liouville cobordism is a cobordism $F$ together with an exact symplectic form $\omega$ on Int $F$ which admits a primitive $\lambda$ such that:

- For some/any function $u: F \rightarrow \mathbb{R}_{\geq 0}$ with regular level set $\partial_{+} F=\{u=0\}$, the product $u \lambda$ extends to a smooth 1 -form on Int $F \cup \partial_{+} F$ which induces a contact form on $\partial_{+} F$.

- For some/any function $u: F \rightarrow \mathbb{R}_{\geq 0}$ with regular level set $\partial_{-} F=\{u=0\}$, the quotient $\lambda / u$ extends to a smooth 1 -form on Int $F \cup \partial_{-} F$ which induces a contact form on $\partial_{-} F$.

Thus, an ideal Liouville domain $(F, \omega)$ is an ideal Liouville cobordism for which $\partial_{-} F$ is empty.

All the results discussed above readily extend to ideal Liouville cobordisms. In particular, both $\partial_{-} F$ and $\partial_{+} F$ inherit canonical contact structures which are positive for their prescribed orientations. In other words, $\partial_{-} F$ is concave while $\partial_{+} F$ is convex.

Finally, the global exactness condition on the symplectic form can be relaxed since exactness is needed only near the boundary. This leads to the definition of ideal symplectic domains/cobordisms. 


\section{B Ideal Liouville domains in contact geometry}

We will now explain how the notion of ideal Liouville domain can help in the study of the relationships between contact structures and open books. We begin with a few basic definitions and constructions.

An open book in a closed manifold $V$ is a pair $(K, \theta)$, where:

- $K \subset V$ is a submanifold of codimension 2 with trivial normal bundle.

- $\theta: V-K \rightarrow \mathbb{S}^{1}=\mathbb{R} / 2 \pi \mathbb{Z}$ is a smooth locally trivial fibration which, in some neighborhood $\mathbb{D}^{2} \times K$ of $K=\{0\} \times K$, is simply the (pullback of the) angular coordinate in $\mathbb{D}^{2}-\{0\}$.

The submanifold $K$ is called the binding of the open book while the closures of the fibers of $\theta$ are the pages. The binding and the pages inherit coorientations from the canonical orientation of $\mathbb{S}^{1}$. Hence, if $V$ is oriented, they are automatically oriented (and the binding is oriented as the boundary of every page).

In practice, most often open books arise from (smooth) complex-valued maps. If a map $h: V \rightarrow \mathbb{C}$ vanishes transversely, with zero-set $K:=\{h=0\}$, and if the argument function $\theta:=h /|h|: V-K \rightarrow \mathbb{S}^{1}$ has no critical points, then the pair $(K, \theta)$ is an open book. Obviously, every open book $(K, \theta)$ can be obtained in this way, and the defining map $h$ is unique up to multiplication by a positive function.

An open book $(K, \theta)$ in a closed manifold $V$ is characterized by its monodromy, which is a diffeomorphism of the 0 -page $F:=K \cup\{\theta=0\}$ relative to the boundary $K$ and defined only up to isotopy. More precisely, consider the affine space of spinning vector fields, namely vector fields $\nu$ on $V$ satisfying the following properties:

- $\nu=0$ along $K$ and $\nu \cdot \theta=2 \pi$ in $V-K$;

- $\nu$ is weakly smooth in the sense that it lifts to a smooth vector field on the manifold with boundary obtained from $V$ by a real oriented blowup along $K$ (see Remark 12 for comments on this condition).

For any such vector field $\nu$, the time 1 map of its flow, restricted to $F$, is a diffeomorphism $\phi$ of $F$ relative to $K$. Moreover, as $\nu$ runs over its affine space, $\phi$ sweeps out an entire mapping class in $\operatorname{MCG}(F):=\pi_{0} \mathcal{D}_{\partial}(F)$ (cf. Remark 12). This mapping class - and sometimes also, by extension, any of its representatives - is the monodromy of the open book $(K, \theta)$. 
Conversely, given a domain $F$ with non-empty boundary and a diffeomorphism $\phi$ of $F$ relative to $K:=\partial F$, one can construct a closed manifold $\mathrm{OB}(F, \phi)$ endowed with an obvious open book whose 0-page is parametrized by $F$ and whose monodromy is represented by $\phi$. There are two steps in the construction.

1) We consider the mapping torus of $\phi$, namely the quotient

$$
\operatorname{MT}(F, \phi):=(\mathbb{R} \times F) / \sim \quad \text { where }(t, p) \sim(t-1, \phi(p)) .
$$

This is a compact manifold (with boundary) which has an obvious fibration

$$
\widehat{\theta}: \operatorname{MT}(F, \phi) \rightarrow \mathbb{S}^{1}=\mathbb{R} / 2 \pi \mathbb{Z}
$$

coming from the projection $\mathbb{R} \times F \rightarrow \mathbb{R}$ multiplied by $2 \pi$. All fibers are diffeomorphic to $F$ and we use the projection $\mathbb{R} \times F \rightarrow \operatorname{MT}(F, \phi)$ restricted to $\{0\} \times F$ as a special parametrization of the 0 -fiber $\{\widehat{\theta}=0\}$ by $F$. We notice that, since $\phi$ induces the identity on $K=\partial F$, the boundary of $\operatorname{MT}(F, \phi)$ is canonically diffeomorphic to $\mathbb{S}^{1} \times K$, the restriction of $\widehat{\theta}$ to $\partial \mathrm{MT}(F, \phi)$ being given by the projection $\mathbb{S}^{1} \times K \rightarrow \mathbb{S}^{1}$.

An important point about the manifold $\operatorname{MT}(F, \phi)$ is that it depends only on the mapping class of $\phi$ in the following sense: If $\phi_{0}$ and $\phi_{1}$ are diffeomorphisms of $F$ relative to $K$ and representing the same mapping class in $\operatorname{MCG}(F)$, then there is a diffeomorphism $\operatorname{MT}\left(F, \phi_{0}\right) \rightarrow \operatorname{MT}\left(F, \phi_{1}\right)$ which respects the fibrations over $\mathbb{S}^{1}$ and the special parametrizations of the 0 -fibers.

2) We construct the closed manifold $\operatorname{OB}(F, \phi)$ from $\operatorname{MT}(F, \phi)$ by collapsing every circle $\mathbb{S}^{1} \times\{.\} \subset \mathbb{S}^{1} \times K=\partial \mathrm{MT}(F, \phi)$ to a point. Thus, $\operatorname{OB}(F, \phi)$ is the union of $\operatorname{Int} \operatorname{MT}(F, \phi)$ and $K=\left(\mathbb{S}^{1} \times K\right) / \mathbb{S}^{1}$. We denote by

$$
\theta: \operatorname{OB}(F, \phi)-K=\operatorname{Int} \operatorname{MT}(F, \phi) \rightarrow \mathbb{S}^{1}
$$

the restriction of the fibration $\widehat{\theta}$.

To see that $(K, \theta)$ is indeed an open book in $\mathrm{OB}(F, \phi)$, we need to specify the smooth structure near $K$. In short, we blow down $\partial \mathrm{MT}(F, \phi)$, the points of $\partial \operatorname{MT}(F, \phi)=\mathbb{S}^{1} \times K$ corresponding to oriented lines in the (trivial) normal bundle of $K$ in $\operatorname{OB}(F, \phi)$. Concretely, we fix a collar neighborhood $\widehat{N}$ of $\partial \operatorname{MT}(F, \phi)$ whose fibers are intervals contained in the fibers of $\widehat{\theta}$ and we declare that, for every $p \in K$, the union of all intervals ending on $\mathbb{S}^{1} \times\{p\} \subset \partial \mathrm{MT}(F, \phi)$ projects to a smooth disk $D_{p}$ in $\operatorname{OB}(F, \phi)$ transverse to $K$ at $p$. More specifically, we choose a function $\widehat{r}: \operatorname{MT}(F, \phi) \rightarrow \mathbb{R}_{\geq 0}$ with regular level set $\partial \operatorname{MT}(F, \phi)=\{\widehat{r}=0\}$, and 
we take the induced function $r$ on $\mathrm{OB}(F, \phi)$, together with $\theta$, as polar coordinates near $p$ on the disk $D_{p}$.

It is not hard to check that a different choice of collar neighborhood $\widehat{N}$ and function $\widehat{r}$ leads to an equivalent smooth structure. Actually, the two structures are conjugated by a homeomorphism of $\operatorname{OB}(F, \phi)$ which preserves $\theta$ and induces the identity on the page $F_{0}:=K \cup\{\theta=0\}$. As a result, $(K, \theta)$ is an open book in $\operatorname{OB}(F, \phi)$, its 0-page $F_{0}$ has a (special) parametrization by $F$, and its monodromy is represented by $\phi$ (note that the vector field $\partial_{t}$ on $\mathbb{R} \times F$ descends to a smooth vector field on $\operatorname{MT}(F, \phi)$, so its image in $\operatorname{OB}(F, \phi)$ is tautologically weakly smooth).

Remark 12 (Smoothly Generated Monodromy Diffeomorphisms). Given an open book $(K, \theta)$ in $V$, one can easily find spinning vector fields $\nu$ on $V$ that are smooth, not just weakly smooth. Thus, the monodromy of $(K, \theta)$ has representatives which are smoothly generated, meaning that they can be obtained by integrating smooth spinning vector fields $\nu$ on $V$. In particular, one can check that any representative of the monodromy which is the identity on a neighborhood of $K$ is smoothly generated (see Lemma 16 for the symplectic version of this assertion). However, Not every representative of the monodromy is smoothly generated. The following simple example was pointed out to me by Roussarie [Ro].

Consider in $\mathbb{R}^{2}$ a smooth vector field $\nu=2 \pi\left(\partial_{\theta}+r f \partial_{r}\right)$, where $f: \mathbb{R}^{2} \rightarrow \mathbb{R}$ is a smooth function. Let $\psi, \phi: \mathbb{R} \rightarrow \mathbb{R}$ denote the diffeomorphisms of the $x$-axis induced by the flow of $\nu$ at times $1 / 2$ and 1 , respectively. Then $\phi$ and $\psi$ commute, and $\psi$ reverses orientation while $\phi$ preserves it. These properties restrict the behavior of $\phi$. For instance, the germ of $\phi$ at 0 cannot have the shape $\phi(x)=x+x^{2}+$ higher order terms. More generally, here is Roussarie's observation: If $\phi-$ id is not infinitely flat at 0 then the first non-zero term in its Taylor expansion has odd degree. Indeed, if $\phi-$ id is not flat, it has a fixed sign on $(0, \varepsilon]$ for $\varepsilon>0$ sufficiently small. Suppose that $\phi(x)>x$ for all $x \in(0, \varepsilon]$. Since $\psi$ is decreasing and commutes with $\phi$,

$$
\phi \circ \psi(x)=\psi \circ \phi(x)<\psi(x) \text { for all } x \in(0, \varepsilon] .
$$

Hence, $\phi(x)<x$ for all $x \in[\psi(\varepsilon), 0)$, and this proves the claim.

In contrast, if the vector field $\nu=2 \pi\left(\partial_{\theta}+r f \partial_{r}\right)$ is only assumed to be "weakly smooth" (namely, if $\nu$ lifts to a smooth vector field on the blownup plane), then its return map $\phi$ on $\mathbb{R}_{\geq 0}$ remains smooth and can freely vary in its mapping class. Indeed, the hypothesis means that $f$ is smooth not as a function on $\mathbb{R}^{2}$ but as a function of the polar coordinates $(r, \theta) \in \mathbb{R}_{\geq 0} \times \mathbb{S}^{1}$. Note also that, since $f$ and $d f$ 
are bounded near $\{0\} \times \mathbb{S}^{1}$, the vector field $\nu$ is Lipschitz. These remarks equally apply to weakly smooth spinning vector fields in any dimension.

The following definition was introduced in [Gi2] to establish formal links between open books and contact structures:

Definition 13 (Open Books and Contact Structures). A contact structure $\xi$ on a closed manifold $V$ is supported by an open book $(K, \theta)$ in $V$ if it admits a Pfaff equation $\alpha$ which is adapted to $(K, \theta)$ in the sense that:

- $\alpha$ induces a positive contact form on $K$, and

- $d \alpha$ induces a positive symplectic form on the fibers of $\theta$.

Orientations here come from the orientation of $V$ defined by $\xi$.

We will show below that an open book supporting a contact structure has some specific geometric structure that we now describe:

Definition 14 (Liouville Open Books). A Liouville open book $\left(K, \theta, \omega_{t}\right)$ in a closed manifold $V$ is an open book $(K, \theta)$ whose pages $F_{t}:=K \cup\{\theta=2 \pi t\}$ are equipped with ideal Liouville structures $\omega_{t}\left(2 \pi t \in \mathbb{S}^{1}\right)$ having primitives $\lambda_{t}$ such that: For some/any map $h: V \rightarrow \mathbb{C}$ defining $(K, \theta)$, the products $|h| \lambda_{t}$ are the restrictions to the fibers $F_{t}-K$ of a global (smooth) 1 -form $\beta$ on $V$. Such a 1 -form $\beta$ is referred to as a binding 1-form (associated with $h$ ), as it indeed ties the forms $\omega_{t}$ about $K$.

In this context, we consider the affine space of weakly smooth spinning vector fields $\nu$ on $V$ satisfying the additional condition that $\nu$ preserves the ideal Liouille structures of pages. This means that the flow of $\nu$, which rotates the open book $(K, \theta)$, preserves the family of forms $\omega_{t}$. Equivalently, $\nu$ spans the kernel of a closed 2-form on $V-K$ which induces $\omega_{t}$ on each page $F_{t}$.

For such a symplectically spinning vector field $\nu$, the time 1 map of its flow restricted to the ideal Liouville page $(F, \omega):=\left(F_{0}, \omega_{0}\right)$ is a symplectic diffeomorphism $\phi$ relative to $K=\partial F$. Moreover, as $\nu$ runs over its affine space, $\phi$ sweeps out a full symplectic mapping class in $\operatorname{MCG}(F, \omega):=\pi_{0} \mathcal{D}_{\partial}(F, \omega)$. This mapping class is the symplectic monodromy of the Liouville open book.

The next lemma shows that the symplectic monodromy of a Liouville open book has representatives which are generated by smooth symplectically spinning vector fields and can be further assumed to be the identity on a neighborhood of $K$. As in the usual (non-Liouville) case, however, not every representative of the symplectic monodromy can be generated in this way. 
Lemma 15 (Binding Forms and Monodromy). Let $\left(K, \theta, \omega_{t}\right)$ be a Liouville open book in a closed manifold $V$, and $h: V \rightarrow \mathbb{C}$ a map defining $(K, \theta)$. For every binding 1-form $\beta$, the vector field $\nu$ on $V-K$ spanning the kernel of $d(\beta /|h|)$ and satisfying $\nu \cdot \theta=2 \pi$ extends to a smooth vector field on $V$ which is zero along $K$. Furthermore, $\beta$ can be chosen so that $\nu$ is 1-periodic near $K$.

Note that binding forms associated with any fixed defining map $h$ constitute an affine space. Another thing to be mentioned here is that, among symplectically spinning vector fields, those associated with binding 1-forms generate exact symplectic diffeomorphisms (see our comment following Proposition 17).

Proof. First observe that $\beta \uparrow_{K}$ is a contact form and defines the (common) boundary contact structure of all ideal Liouville pages. We fix a small $\varepsilon>0$ such that $\beta$ induces a contact form on every fiber $K_{w}:=\{h=w\},|w| \leq \varepsilon$. We set $\alpha_{w}:=\beta \uparrow_{K_{w}}$ and $N:=\{|h| \leq \varepsilon\}$. The hyperplane field $\tau:=\operatorname{Ker}\left(\beta \uparrow_{N}\right)$ splits as a direct sum $\tau=\xi \oplus \xi^{\perp}$, where $\xi$ is the subdistribution consisting of the contact structures $\xi_{w}:=\operatorname{Ker} \alpha_{w},|w| \leq \varepsilon$, and $\xi^{\perp}$ is the $d \beta$-orthogonal complement of $\xi$ in $\tau$ (and determines a contact connection over $\varepsilon \mathbb{D}^{2}$ ). Now consider the following vector fields on $N$ :

- $\partial_{\alpha}$ is the vector field in Ker $d h$ whose restriction to each fiber $K_{w}$ of $h$ is the Reeb field $\partial_{\alpha_{w}}$ of $\alpha_{w}$.

- $\widetilde{\partial}_{\theta}$ and $\widetilde{\partial}_{r}$ are the vector fields in $\xi^{\perp}$ projecting to $\partial_{\theta}$ and $\partial_{r}$, respectively, where $(r, \theta)$ denote polar coordinates in $\varepsilon \mathbb{D}^{2}$.

A routine calculation shows that the vector field $\nu$ on $V-K$ spanning the kernel of $d(\beta /|h|)$ and satisfying $\nu \cdot \theta=2 \pi$ is given in $N$ by

$$
\nu=2 \pi\left(\widetilde{\partial}_{\theta}+a r \widetilde{\partial}_{r}+b \partial_{\alpha}\right),
$$

where $r=r \circ h=|h|$ while

$$
a:=\frac{d \beta\left(\widetilde{\partial}_{\theta}, \partial_{\alpha}\right)}{1+d \beta\left(\partial_{\alpha}, r \widetilde{\partial}_{r}\right)} \quad \text { and } \quad b:=\frac{d \beta\left(r \widetilde{\partial}_{r}, \widetilde{\partial}_{\theta}\right)}{1+d \beta\left(\partial_{\alpha}, r \widetilde{\partial}_{r}\right)}
$$

Clearly, $a$ and $b$ are smooth functions on $N$ and vanish identically along $K$, so $\nu$ has the desired smooth extension on $V$.

We will now modify $\beta$ to obtain a binding form $\beta^{\prime}$ such that the spinning vector field $\nu^{\prime}$ spanning the kernel of $d\left(\beta^{\prime} /|h|\right)$ in $V-K$ is 1-periodic near $K$. 
First, we trivialize $N$ as a product $N=\varepsilon \mathbb{D}^{2} \times K$ so that, in the corresponding cylindrical coordinates $(r, \theta, q)$, the vector field $\partial_{r}$ lies in $\xi^{\perp}$. In other words, $\partial_{r}$ equals $\widetilde{\partial}_{r}$ and, along $K=\{0\} \times K$, the 2-plane field $\xi^{\perp}$ is horizontal (namely, tangent to the disks $\mathbb{D}^{2} \times\{q\}, q \in K$ ). It is then an exercise to check that $\beta\left(\partial_{\theta}\right) d \theta$ is a smooth form in $N$. Now pick a function $\rho: V \rightarrow[0,1]$ compactly supported in $N$ and equal to 1 near $K$, and let

$$
\beta^{\prime}:=\beta-\rho \beta\left(\partial_{\theta}\right) d \theta
$$

This smooth 1-form on $V$ coincides with $\beta$ on every page, so it is a binding form. Moreover, near $K$,

$$
\beta^{\prime}=\beta-\beta\left(\partial_{\theta}\right) d \theta=f \pi^{*} \alpha_{0}
$$

where $f$ is a positive function, $\pi$ the projection $N=\varepsilon \mathbb{D}^{2} \times K \rightarrow K$ and $\alpha_{0}$ the restriction of $\beta$ to $K$. It follows that the spinning vector field $\nu^{\prime}$ spanning the kernel of $d\left(\beta^{\prime} /|h|\right)$ is horizontal (in the product structure of $N$ ) and tangent to the level sets of the function $f /|h|$. Therefore, $\nu^{\prime}$ is 1-periodic.

A practical consequence of this lemma is:

Lemma 16 (Criterion for Smooth Generation). Any representative of the symplectic monodromy of a Liouville open book which is the identity near the boundary is generated by a smooth symplectically spinning vector field.

Proof. This follows from the last assertion of Lemma 15 and the fact that, if two symplectic diffeomorphisms of an ideal Liouville domain $(F, \omega)$ coincide with the identity near $K:=\partial F$ and represent the same class in $\operatorname{MCG}(F, \omega)$, then they are connected by a symplectic isotopy relative to a neighborhood of $K$ (an easy way to construct such an isotopy is to use the embeddings of Proposition 3 as in the proof of Corollary [5).

The next proposition is a variation on a wellknown construction first introduced by Thurston-Winkelnkemper in three dimensions [TW] and extended to higher dimensions in [Gi2]:

Proposition 17 (Construction of Liouville Open Books). Consider an ideal Liouville domain $(F, \omega)$ and a symplectic diffeomorphism $\phi: F \rightarrow F$ relative to $K:=\partial F$. The open book in $\mathrm{OB}(F, \phi)$ is a Liouville open book for which the parametrization of its 0-page by $F$ is a symplectomorphism. 
The proof below actually shows that, if the symplectic diffeomorphism $\phi$ is the identity near $K$ and is exact (meaning that there exists an ideal Liouville form $\lambda$ such that $\phi^{*} \lambda-\lambda$ is the differential of a function with compact support in Int $F$ ), then $\phi$ is (smoothly) generated by the spinning vector field of a binding 1-form.

Proof. Let $\lambda_{t}$ be a path of ideal Liouville forms on $(F, \omega)$ joining an arbitrary $\lambda_{0}$ to $\lambda_{1}:=\phi^{*} \lambda_{0}$. According to Corollary 5, there is a symplectic isotopy $\psi_{t}$ of $F$, relative to $K$, such that $\psi_{0}=$ id and $\psi_{t}^{*} \lambda_{t}-\lambda_{0}=d f_{t}$ for all $t \in[0,1]$, where the functions $f_{t}$ have compact supports in Int $F$. Then the symplectic isotopy $\phi_{t}:=\phi \circ \psi_{t}$ is relative to $K$ and connects $\phi=\phi_{0}$ to a symplectic diffeomorphism $\phi_{1}$ which is exact and coincides with the identity near $K$. Since $\operatorname{OB}(F, \phi)$ depends only on the (smooth) mapping class of $\phi$, we assume from now on that $\phi$ is exact and is the identity on a neighborhood of $K$.

We now pick an ideal Liouville form $\lambda$ such that $\phi^{*} \lambda=\lambda+d f_{1}$, where $f_{1}$ is a function with compact support in Int $F$, and we choose a path of functions $f_{t}$ - all with compact supports in Int $F$ - joining $f_{0}:=0$ to $f_{1}$. Then the 1 -form $\lambda+d f_{t}+\dot{f}_{t} d t$ on $[0,1] \times \operatorname{Int} F$ is a primitive of (the pullback of) $\omega$ and descends to a 1 -form $\widehat{\beta}$ on $\operatorname{Int} \operatorname{MT}(F, \phi)$ (to ignore smoothing issues, take the path $f_{t}$ to be constant near its endpoints).

The next step is to fix cylindrical coordinates near $K$ in $\mathrm{OB}(F, \phi)$ and a map $h$ : $\mathrm{OB}(F, \phi)$ defining the obvious open book. We pick a non-negative function $u$ on $F$, with regular level set $K=\{u=0\}$, such that:

- $\vec{\lambda} \cdot \log u=-1$ in a neighborhood of $K$ (equivalently, the Lie derivative $\vec{\lambda} \cdot(u \lambda)$ is zero), and

- $u \circ \phi=u$ (this property is typically satisfied if $u$ is constant on the support of $\phi$ ).

Then the map

$$
(t, p) \in[0,1] \times F \mapsto u(p) e^{2 i \pi t} \in \mathbb{C}
$$

provides the required defining map $h: \mathrm{OB}(F, \phi) \rightarrow \mathbb{C}$. Furthermore, the function $u$ and the collar neighborhood of $K$ associated with $\lambda$ (cf. Proposition 3) provide cylindrical coordinates near $K$. More precisely, let $G:=\{u \leq \varepsilon\} \subset F$ with $\varepsilon$ small enough that $\lambda \cdot \log u=-1$ on $G$ and $G$ is disjoint from the supports of $\phi$ and of all functions $f_{t}(t \in[0,1])$. Then the function $u$ and the foliation spanned by $\lambda$ identify $G$ with $[0, \varepsilon] \times K$. In the same way, $N:=\{|h| \leq \varepsilon\}$ is identified with $\varepsilon \mathbb{D}^{2} \times K$. 
It remains to see that the form $|h| \widehat{\beta}$ on $\operatorname{OB}(F, \phi)-K$ extends to a smooth (binding) form on $\operatorname{OB}(F, \phi)$. In fact, the form $\left.u \lambda\right|_{G-K}$ is invariant under the flow of $\lambda$, so it is the pullback on $(0, \varepsilon] \times K$ of a 1-form $\alpha$ on $K$. Similarly, the form $\left.|h| \widehat{\beta}\right|_{N-K}$ is the pullback on $\left(\varepsilon \mathbb{D}^{2}-\{0\}\right) \times K$ of the same 1 -form $\alpha$ on $K$. Hence, it extends smoothly across $K$.

Now the most obvious relationship between supporting and Liouville open books is:

Proposition 18 (Supporting Open Book are Liouville). Let $(V, \xi)$ be a closed contact manifold, and $(K, \theta)$ a supporting open book with defining map $h: V \rightarrow \mathbb{C}$. Then the equations $\alpha$ of $\xi$ such that $d(\alpha /|h|)$ induces an ideal Liouville structure on each page form a non-empty convex cone.

Proof. This follows readily from uniqueness of ideal completions of (usual) Liouville domains (see Example 9).

An equation $\alpha$ of $\xi$ as in the above proposition yields ideal Liouville structures $\omega_{t}$ on the fibers of $\theta$, and $\left(K, \theta, \omega_{t}\right)$ is a Liouville open book. By Lemma 15, the kernel of $d(\alpha /|h|)$ is spanned by a smooth symplectically spinning vector field $\nu$, but it is easy to verify that $\nu$ is never 1 -periodic near $K$. Though it may create some psychological disconfort, this inconvenience is not a problem. It could in fact be remedied by replacing Liouville open books with open books whose pages are given "degenerate ideal Liouville structures" (to define those objects, take Definition 1 and simply substitute $u \lambda$ with $u^{2} \lambda$ in the extension condition). In short, the key observation here is that, if we consider for instance the contact form $\alpha:=d z+r^{2} d \theta$ in 3 -space (with cylindrical coordinates $(r, \theta, z)$ ) then, away from the $z$-axis, the Reeb field of $\alpha / r^{2}$ is $\partial_{\theta}$ while the Reeb field of $\alpha / r$ is proportional to the vector field $\partial_{\theta}+r^{2} \partial_{z}$.

Proposition 18 leads to a new definition:

Definition 19 (Liouville Open Books and Contact Structures). Let $\left(K, \theta, \omega_{t}\right)$ be a Liouville open book on a closed manifold $V$, and $h: V \rightarrow \mathbb{C}$ a map defining $(K, \theta)$. A contact structure on $V$ is (symplectically) supported by $\left(K, \theta, \omega_{t}\right)$ if it admits a binding equation on $V$, that is, an equation $\alpha$ such that $\alpha /|h|$ induces an ideal Liouville form on each ideal Liouville page $\left(F_{t}, \omega_{t}\right)\left(2 \pi t \in \mathbb{S}^{1}\right)$.

Remark 20 (Uniqueness of the Binding Equation). If it exists, the above equation $\alpha$ is unique (the defining map $h$ being fixed). The underlying more general assertion is that, given an ideal Liouville domain $(F, \omega)$ and an ideal Liouville form $\lambda$, 
the constant function 1 is the only function $f$ on Int $F$ such that $d(f \lambda)=\omega$. For $\operatorname{dim} F \geq 4$, the reason is purely algebraic: $f \omega$ and $\omega$ must agree on the kernel of $\lambda$, which contains an $\omega$-symplectic space; hence $f$ has to equal 1 . If $\operatorname{dim} F=2$, non-constant solutions $f$ exist locally, so we need a more global argument. Since

$$
d(f \lambda)=f \omega+d f \wedge \lambda=(f+\underline{\lambda} \cdot f) \omega,
$$

the condition $d(f \lambda)=\omega$ reads $g+\lambda \vec{A} \cdot g=0$, where $g:=f-1$. Now any nonzero solution $g$ of this equation has to be unbounded on every complete non-trivial orbit of $\lambda$. The claim then follows from $\lambda$ being complete (Proposition 3 ).

If a contact structure is (symplectically) supported by a Liouville open book then it is supported by the underlying smooth open book: To obtain an adapted equation in the sense of Definition [13, simply replace $\alpha /|h|$ by $\alpha / u(|h|)$ where $u: \mathbb{R}_{\geq 0} \rightarrow \mathbb{R}_{>0}$ is an increasing function such that $u(x)=x$ for $x \geq \varepsilon$ and $u(x)=x^{2}+\varepsilon^{2}$ for $x \leq \varepsilon / 2$ (with $\varepsilon$ sufficiently small).

We now conclude this paper by showing that the inclusion of the space of contact structures supported by a Liouville open book into the affine space of binding forms is a weak homotopy equivalence:

Proposition 21 (Existence and Uniqueness of Supported Contact Structures). On a closed manifold, contact structures supported by a given Liouville open book form a non-empty and weakly contractible subset in the space of all contact structures. In particular, they lie in a unique isotopy class.

Note that the symplectic orientation of the pages, together with their natural coorientation, determines an orientation of the ambient manifold. It is implicit in this statement that the supported contact structures are positive for this orientation.

Proof. Let $V$ be the ambient closed $2 n+1$-manifold, $\left(K, \theta, \omega_{t}\right)$ a Liouville open book in $V$ and $h: V \rightarrow \mathbb{C}$ a map defining $(K, \theta)$. For any binding form $\beta$ on $V$ (associated with $h$ ), we can find an $\varepsilon>0$ such that $\beta$ induces a contact form on every fiber $K_{w}:=\{h=w\}$ with $|w| \leq \varepsilon$. We fix a non-decreasing function $f: \mathbb{R}_{\geq 0} \rightarrow \mathbb{R}$ such that $f(x)=x$ for $x \leq \varepsilon / 2$ and $f(x)=1$ for $x \geq \varepsilon$. Then, for $c \geq 0$, we define

$$
\beta_{c}:=\beta+c|h| f(|h|) d \theta .
$$

Clearly, $\beta_{c} /|h|$ coincides with $\beta /|h|$ on every page. Therefore, if $\beta_{c}$ is a contact form, the contact structure it defines is symplectically supported by our Liouville open book. We claim that $\beta_{c}$ is a contact form for all sufficiently large $c$, and in 
fact for all $c \geq 0$ if $\beta$ itself is already a contact form. To see this, we set $r:=|h|$ and we write

$$
\begin{aligned}
\beta_{c} \wedge\left(d \beta_{c}\right)^{n}=n c r f^{\prime}(r) d r & \wedge d \theta \wedge \beta \wedge(d \beta)^{n-1} \\
+c f(r) d \theta & \wedge(r d \beta+n \beta \wedge d r) \wedge(d \beta)^{n-1}+\beta \wedge(d \beta)^{n} .
\end{aligned}
$$

Since $\beta$ induces a contact form on each fiber $K_{w}$ of $h$ with $|w| \leq \varepsilon$, the term $r f^{\prime}(r) d r \wedge d \theta \wedge \beta \wedge(d \beta)^{n-1}$ is a positive volume form provided $f^{\prime}(r) \neq 0$. On the other hand, for all $r>0$,

$$
f(r) d \theta \wedge(r d \beta+n \beta \wedge d r) \wedge(d \beta)^{n-1}=r^{n+1} f(r) d \theta \wedge(d(\beta / r))^{n}
$$

is also a positive volume form. The claim follows.

Now consider a $k$-sphere $\xi_{s}, s \in \mathbb{S}^{k}$, of contact structures supported by the Liouville open book $\left(K, \theta, \omega_{t}\right)$. By Remark 20, every contact structure $\xi_{s}$ has a unique binding equation $\alpha_{s}$, and the forms $\alpha_{s}, s \in \mathbb{S}^{k}$, depend continuously on $s$. Since binding forms (associated with a fixed $h$ ) constitute an affine space, we can find a $(k+1)$-disk of binding forms $\beta_{s}, s \in \mathbb{D}^{k+1}$, such that $\beta_{s}=\alpha_{s}$ for all $s \in \mathbb{S}^{k}=\partial \mathbb{D}^{k+1}$. We choose $\varepsilon>0$ small enough that each $\beta_{s}, s \in \mathbb{D}^{k+1}$, induces a contact form on all fibers $K_{w}$ with $|w| \leq \varepsilon$, and we apply our claim twice:

- For some $c_{0}$ sufficiently large, the forms

$$
\beta_{s, c_{0}}:=\beta_{s}+c_{0}|h| f(|h|) d \theta, \quad s \in \mathbb{D}^{k+1},
$$

constitute a $(k+1)$-disk of contact forms.

- For the same value $c_{0}$, the forms

$$
\alpha_{s, c}:=\alpha_{s}+c|h| f(|h|) d \theta, \quad s \in \mathbb{S}^{k}, c \in\left[0, c_{0}\right],
$$

constitute a homotopy of $k$-spheres of contact forms between the original $k$-sphere $\alpha_{s}=\alpha_{s, 0}, s \in \mathbb{S}^{k}$, and the $k$-sphere $\alpha_{s, c_{0}}=\beta_{s, c_{0}}, s \in \mathbb{S}^{k}$, which bounds a $(k+1)$-disk of contact forms.

Since all these contact forms are binding forms, all the contact structures they define are supported by the Liouville open book $\left(K, \theta, \omega_{t}\right)$, and so our argument shows that the $k$-sphere $\xi_{s}, s \in \mathbb{S}^{k}$, is nulhomotopic in the space of contact structures supported by $\left(K, \theta, \omega_{t}\right)$. 


\section{References}

[BEE] F. Bourgeois, T. EKholm and Y.EliAshberG - Effect of Legendrian surgery. Geom. Topol. 16 (2012), 301-389.

[CE] K. CieliebaK and Y. Eliashberg - From Stein to Weinstein and Back. Amer. Math. Soc. Colloq. Publ. 59, Amer. Math. Soc. 2012.

[CFH] K. Cieliebak, A. Floer and H. Hofer - Symplectic homology, II: A general construction. Math. Z. 218 (1995), 103-122.

[Co1] S. COURTE - Contact manifolds with symplectomorphic symplectizations. Geom. Topol. 18 (2014), 1-15.

[Co2] S. COURTE - h-Cobordismes en Géométrie Symplectique. Thèse de Doctorat, École Normale Supérieure de Lyon, juin 2015.

[Do] S. DonaldSON - Symplectic submanifolds and almost-complex geometry. J. Diff. Geom. 44 (1996), 666-705.

[EG] Y. Eliashberg and M. Gromov - Convex symplectic manifolds. Several Complex Variables and Complex Geometry, part 2, 135-162, Proc. Sympos. Pure Math. 52, Amer. Math. Soc. 1991.

[EKP] Y. EliashberG, S. S. Kim and L. Polterovich - Geometry of contact transformations and domains: Orderability versus squeezing. Geom. Topol. 10 (2006), 1635-1747.

[Ge] H. GEIGES - Symplectic manifolds with disconnected boundary of contact type. Internat. Math. Res. Notices (1994), 23-30.

[Gi1] E. Giroux - Convexité en topologie de contact. Comment. Math. Helv. 66 (1991), 637-677.

[Gi2] E. GIROUX - Géométrie de contact : de la dimension trois vers les dimensions supérieures. Proceedings of the International Congress of Mathematicians, vol. 2, 405-414, Higher Ed. Press 2002.

[La] F. LAUDENBACH - Engouffrement symplectique et intersections lagrangiennes. Comment. Math. Helv. 70 (1995), 558-614. 
[MNW] P. Massot, K. NiederkrüGer and C. WendL - Weak and strong fillability of higher dimensional contact manifolds. Invent. Math. 192 (2013), 287-373.

[Mc] D. MCDuFF - Symplectic manifolds with contact type boundaries. Invent. Math. 103 (1991), 651-671.

[Ro] R. RouSSARIE — Private communication by e-mail, July 2017.

[Se] P. SEIDEL - A biased view of symplectic cohomology. Current Developments in Mathematics, 211-253, Int. Press 2008.

[TW] W. THURSTON and H. WINKELNKEMPER - On the existence of contact forms. Proc. Amer. Math. Soc. 52 (1975), 345-347.

[Vi] C. Viterbo - Functors and computations in Floer homology with applications, I. Geom. Funct. Anal. 9 (1999), 985-1033.

[We] A. WeINSTEIN - On the hypotheses of Rabinowitz' periodic orbit theorems. J. Differential Equations 33 (1979), 353-358. 